IFUM-824-FT

\title{
Slavnov-Taylor parameterization of Yang-Mills theory with massive fermions in the presence of singlet axial-vector currents
}

\author{
Andrea Quadri ${ }^{1}$ \\ Dip. di Fisica, Università di Milano, via Celoria 16, 20133 Milano, Italy \\ and I.N.F.N., sezione di Milano
}

\begin{abstract}
We study the all-order restoration of the Slavnov-Taylor (ST) identities for Yang-Mills theory with massive fermions in the presence of singlet axial-vector currents. By making use of the ST parameterization of the symmetric quantum effective action a natural set of normalization conditions is derived allowing to reduce the algebraic complexity of higher orders ST identities up to a homogeneous linear problem. Explicit formulas for the action-like part of the symmetric vertex functional are given to all orders in the loop expansion.
\end{abstract}

\footnotetext{
${ }^{1}$ E-mail address: andrea.quadri@mi.infn.it
} 


\section{Introduction}

In the BRST quantization [1- [7] of gauge theories the requirement of Physical Unitarity is guaranteed by the fulfillment of the Slavnov-Taylor (ST) identities [8, 9].

Even in the absence of anomalies the ST identities may be spoiled by the regularization procedure used to remove UV divergences in the Feynman amplitudes. For theories involving the $\gamma^{5}$ matrix and the completely antisymmetric tensor dimensional regularization is not an invariant regularization scheme. An invariant regularization for anomaly-free chiral gauge theories like the Standard Model and the $\mathrm{SO}(10)$ chiral theory has been introduced in [10]-[11]. On the other hand, in the context of supersymmetric theories no all-order invariant regularization scheme is known.

By exploiting cohomological techniques it can be proven in a regularizationindependent way that, if the model is anomaly-free, an order-by-order choice of non-invariant counterterms exists allowing to recursively fulfill the ST identities. Along these lines the Standard Model (SM) has been dealt with in [15, 16, 17. and the Minimal Supersymmetric Standard Model in [18.

On the other hand, there is no completely satisfactory answer to the question of how to find the explicit form of the non-invariant symmetry-restoring counterterms. Modified subtraction schemes have been considered e.g. in 33[36. Methods based on the computation of the ST breaking terms have been applied in [19] and 20]. Within the framework of Algebraic Renormalization [7. the direct imposition of the ST identities after expansion of the symmetric vertex functional on Lorentz-invariant monomials, compatible with all unbroken symmetris of the model, has been proposed in 21] and applied to some specific SM examples in [22]-24]. In [25] simplifications coming from the use of the background field method have also been discussed. Proper subsets of relations between Green functions, stemming from the ST identities, have been considered for several supersymmetric models in [26]-[28].

In the absence of IR problems an alternative proposal was suggested in 30. The finite terms of the action-like part of $\mathbb{\Gamma}$ are constructively parameterized by making use of the elements of the cohomology of the classical linearized ST operator $\mathcal{S}_{0}$ without power-counting restrictions (ST invariants) order by order in the loop expansion. This in turn allows for a systematic explicit derivation of the non-invariant counterterms recursively needed in order to restore the ST identities.

The purpose of the present paper is to apply such a technique to the study of Yang-Mills theory with massive fermions in the presence of singlet axial-vector currents.

This model is directly related to the QCD sector of the Standard Model. There the singlet axial-vector currents characterize the couplings of the neutral vector bosons to the quarks. Two-loop QCD corrections to the heavy quark form factors have been recently discussed in the framework of dimensional regularization in 31, 32.

Here we choose to work with the non-invariant BPHZL procedure in order 
to regularize the Feynman amplitudes. The ST parameterization is constructed for the model at hand and used to study the properties of the action-like part of the symmetric quantum effective action, i.e. the projection of the 1-PI vertex functional onto the sector spanned by all possible Lorentz-invariant monomials of dimension $\leq 4$ in the fields, the antifields, the external sources and their derivatives, compatible with all unbroken symmetries of the theory.

When cohomologically non-trivial masses (unlike IR regulators) are present, the finite $n$-th order non-symmetric action-like terms depend on superficially convergent $n$-th order Feynman amplitudes. This property has been pointed out for the Abelian Higgs-Kibble model in [21. By using the ST parameterization, the underlying geometrical structure is investigated and clarified here in a full non-Abelian setting.

In 21] it was also noted that, due to the presence of physical mass parameters, a number of consistency conditions involving superficially convergent Feynman amplitudes appears. The origin of these relations can be traced back to the Wess-Zumino consistency conditions for the ST breaking terms [21. This becomes particularly clear when ST invariants are used in order to parameterize the symmetric quantum effective action. This issue is illustrated on the non-trivial set of consistency conditions arising in the present model.

At orders higher than one the ST identities are intrinsically non-linear. Nevertheless, by using the ST parameterization of the quantum effective action it can be proven that in the model under investigation there exists a special choice of normalization conditions such that the relevant action-like part of the symmetric quantum effective action obeys a linear identity also at higher orders. The existence of this special set of normalization conditions is related to the geometrical structure of the non-invariant action-like terms.

By imposing these normalization conditions explicit formulas for the actionlike part of the symmetric vertex functional are finally derived to all orders in the loop expansion.

We remark that the inclusion of composite operators like the singlet axialvector currents does not pose any additional problem in the application of the ST parameterization technique. Hence the latter also provides an efficient way to treat BRST-invariant composite operators in those theories where no invariant regularization scheme is known.

The paper is organized as follows. In Sect.2 the classical action of Yang-Mills theory with massive fermions in the presence of singlet axial-vector currents is considered in the BPHZL scheme. The IR regulator for gauge fields and ghosts is cast in a form useful for dealing with BRST symmetry along the lines of [37. In Sect. 3] the ST parameterization of the quantum effective action is used in order to derive the most general form of the action-like part of the symmetric vertex functional. The fulfillment of the ST identities implies a set of relations involving only superficially convergent Feynman amplitudes. In Sect. 4 we show that these relations actually hold true as a consequence of the Wess-Zumino consistency condition. In Sect. 5 the $n$-th order ST identities are analyzed and a natural set of normalization conditions is obtained, leading to the linearization 
of the ST identity obeyed by the action-like part of the $n$-th order symmetric vertex functional. Finally conclusions are presented in Sect. [6

\section{The model}

We consider a semi-simple gauge group $G$ and denote by $T^{a}$ the hermitean generators of the associated Lie algebra $\mathfrak{g}$ in the fermionic matter representation. The generators $T^{a}$ obey the commutation relation

$$
\left[T^{a}, T^{b}\right]=i f^{a b c} T^{c}
$$

where $f^{a b c}$ are the structure constants of $\mathfrak{g}$.

We wish to study the model described by the action:

$$
\begin{gathered}
S=\int d^{4} x\left(-\frac{1}{4 g^{2}} F_{\mu \nu}^{a} F^{\mu \nu a}+i \bar{\psi}_{i} \not \supset \psi_{i}-M \bar{\psi}_{i} \psi_{i}\right. \\
\left.+\beta_{\mu}^{V} \bar{\psi} \gamma^{\mu} \psi+\beta_{\mu}^{A} \bar{\psi} \gamma^{\mu} \gamma^{5} \psi\right),
\end{gathered}
$$

invariant under the following infinitesimal gauge transformations of parameters $\alpha^{a}$ :

$$
\begin{aligned}
& \delta A_{\mu}^{a}=\left(D_{\mu} \alpha\right)^{a} \equiv \partial_{\mu} \alpha^{a}+f^{a b c} A_{\mu}^{b} \alpha^{c}, \\
& \delta \psi_{i}=i \alpha^{a} T^{a} \psi_{i}, \quad \delta \bar{\psi}_{i}=-i \alpha^{a} \bar{\psi}_{i} T^{a} .
\end{aligned}
$$

In eq.(2) $g$ denotes the Yang-Mills coupling constant. $F_{\mu \nu}^{a}$ stands for the field strength of the gauge fields $A_{\mu}^{a}$ :

$$
F_{\mu \nu}^{a}=\partial_{\mu} A_{\nu}^{a}-\partial_{\nu} A_{\mu}^{a}+f^{a b c} A_{\mu}^{b} A_{\nu}^{c} .
$$

The covariant derivative on the fermionic fields $\psi_{i}$ is defined by

$$
D_{\mu} \psi_{i}=\partial_{\mu} \psi_{i}-i A_{\mu}^{a} T^{a} \psi_{i}
$$

$\beta_{\mu}^{V}$ is an external source coupled to the singlet vector current $j_{V}^{\mu}=\bar{\psi} \gamma^{\mu} \psi$ while the external source $\beta_{\mu}^{A}$ is coupled to the singlet axial current $j_{A}^{\mu}=\bar{\psi} \gamma^{\mu} \gamma^{5} \psi$.

The BRST transformations of $A_{\mu}^{a}$ and $\psi_{i}, \bar{\psi}_{i}$ are obtained in the usual way by replacing in the gauge transformations of eq. (3) the gauge parameters $\alpha^{a}$ by the anticommuting ghost fields $\omega^{a}$. Nilpotency of the BRST differential $s$ fixes the BRST transformation of $\omega^{a}$. The action of $s$ on $A_{\mu}^{a}, \bar{\psi}_{i}, \psi_{i}$ and $\omega^{a}$ is given in Appendix $\$$ The external sources $\beta_{\mu}^{V}$ and $\beta_{\mu}^{A}$ are BRST-invariant. $S$ in eq.(2) is invariant under $s$.

In order to fix the gauge we choose a standard Lorentz-covariant gauge-fixing condition

$$
F^{a}=\partial A^{a},
$$


which we implement in the BRST formalism by introducing the antighost fields $\bar{\omega}^{a}$ and the corresponding Nakanishi-Lautrup multiplier fields $B^{a}$, with the following BRST transformations:

$$
s \bar{\omega}^{a}=B^{a}, \quad s B^{a}=0 .
$$

Then the gauge-fixing term is

$$
\begin{aligned}
S_{\text {g.f. }} & =\int d^{4} x s\left(\bar{\omega}^{a}\left(\alpha \frac{B^{a}}{2}-\partial A^{a}\right)\right) \\
& =\int d^{4} x\left(\alpha \frac{\left(B^{a}\right)^{2}}{2}-B^{a} \partial A^{a}+\bar{\omega}^{a} \partial^{\mu}\left(D_{\mu} \omega\right)^{a}\right) .
\end{aligned}
$$

$\alpha$ is the gauge parameter. Since the BRST transformations of $A_{\mu}^{a}, \psi_{i}, \bar{\psi}_{i}$ and $\omega^{a}$ are non-linear in the quantum fields, their antifields [7] have to be introduced. We denote them respectively by $A_{\mu}^{a *}, \bar{Y}_{i}, Y_{i}$ and $\omega^{a *}$. The antifield-dependent part of the classical action is obtained by coupling the antifields to the BRST variation of the corresponding fields, as follows:

$$
\begin{aligned}
S_{\text {a.f. }}=\int d^{4} x & \left(A_{\mu}^{a *}\left(D^{\mu} \omega\right)^{a}+\omega^{a *}\left(-\frac{1}{2} f^{a b c} \omega^{b} \omega^{c}\right)\right. \\
& \left.-i \bar{\psi}_{i} T^{a} \omega^{a} Y_{i}+i \bar{Y}_{i} \omega^{a} T^{a} \psi_{i}\right) .
\end{aligned}
$$

The full gauge-fixed BRST-invariant classical action is finally given by

$$
\Gamma^{(0)}=S+S_{\text {g.f. }}+S_{\text {a.f. }}
$$

\subsection{IR regulator in the BPHZL scheme}

In the BPHZL regularization scheme 38-43 massless propagators are handled by the Lowenstein-Zimmermann prescription [41- 43]. An infrared regulator is introduced by assigning to all massless particles an intermediate mass

$$
m^{2}=\mu^{2}(s-1)^{2}
$$

The IR regulator $m^{2}$ depends on the auxiliary parameter $s$ ranging between 0 and 1 while $\mu$ is a fixed constant mass parameter. The subtraction operator $t_{\gamma}$ for a given divergent 1-PI graph or subgraph $\gamma$ involves both a subtraction around $p=0, s=0$ and around $p=0, s=1$ [41]- 43]:

$$
\left(1-t_{\gamma}\right)=\left(1-t_{p, s-1}^{\rho(\gamma)-1}\right)\left(1-t_{p, s}^{\delta(\gamma)}\right)
$$

where $\rho(\gamma)$ is the IR subtraction degree and $\delta(\gamma)$ the UV subtraction degree for $\gamma$ 41]-43. We point out that both subtractions around $s=0$ and $s=1$ are needed in order to guarantee the absence of IR singularities of the 1-PI Green functions in the physical limit $s \rightarrow 1(m \rightarrow 0)$, under the assumption that the IR power-counting criteria of [41-43] are fulfilled. 
The interplay between the ST identities and the BPHZL IR regulator for massless gauge fields and ghosts [44] has been discussed from a cohomological point of view in 37. The mass $m$ in eq. (11) is paired within a BRST doublet with a constant anticommuting parameter $\bar{\rho}$ in such a way that

$$
s \bar{\rho}=m^{2}, \quad s m^{2}=0 .
$$

Notice that each factor $(s-1)$ (and hence $m$ ) in eq.(11) is assigned UV dimension one in the BPHZL subtraction scheme 42 (unlike the fermionic mass $M$, which has UV dimension zero due to the fact that no subtraction in $M$ is performed). The UV dimension of $\bar{\rho}$ is equal to one.

Then the following BRST-invariant mass term is added to the action $\Gamma^{(0)}$ in eq. (10):

$$
\begin{gathered}
s \int d^{4} x\left(\frac{1}{2} \bar{\rho}\left(A_{\mu}^{a}\right)^{2}+\bar{\rho} \bar{\omega}^{a} \omega^{a}\right)=\int d^{4} x\left(\frac{1}{2} m^{2}\left(A_{\mu}^{a}\right)^{2}+m^{2} \bar{\omega}^{a} \omega^{a}\right. \\
\left.-\bar{\rho} A_{\mu}^{a} \partial^{\mu} \omega^{a}-\bar{\rho} B^{a} \omega^{a}-\frac{1}{2} \bar{\rho} \bar{\omega}^{a} f^{a b c} \omega^{b} \omega^{c}\right) .
\end{gathered}
$$

The cohomology of the original BRST differential is unaltered by the inclusion of the BRST doublet $\left(\bar{\rho}, m^{2}\right)$. Accordingly the mass term in eq. (14) is BRSTexact. In the physical limit $m \rightarrow 0, \bar{\rho} \rightarrow 0$ all the invariants of the BRST differential, extended according to eq. (13), reduce to those of the original BRST transformation.

The IR-regulated classical action $\Gamma_{m}^{(0)}$ is reported in Appendix $\mathrm{A}$ The ST identities for $\Gamma_{m}^{(0)}$ become

$$
\begin{aligned}
\mathcal{S}\left(\Gamma_{m}^{(0)}\right)=\int d^{4} x & \left(\frac{\delta \Gamma_{m}^{(0)}}{\delta A_{\mu}^{a *}} \frac{\delta \Gamma_{m}^{(0)}}{\delta A^{a \mu}}+\frac{\delta \Gamma_{m}^{(0)}}{\delta \omega^{a *}} \frac{\delta \Gamma_{m}^{(0)}}{\delta \omega^{a}}+B^{a} \frac{\delta \Gamma_{m}^{(0)}}{\delta \bar{\omega}^{a}}\right. \\
& \left.-\frac{\delta \Gamma_{m}^{(0)}}{\delta Y_{i}} \frac{\delta \Gamma_{m}^{(0)}}{\delta \bar{\psi}_{i}}+\frac{\delta \Gamma_{m}^{(0)}}{\delta \bar{Y}_{i}} \frac{\delta \Gamma_{m}^{(0)}}{\delta \psi_{i}}\right)+m^{2} \frac{\partial \Gamma_{m}^{(0)}}{\partial \bar{\rho}}=0 .
\end{aligned}
$$

The last term in the above equation parameterizes the soft-breaking of the original ST identities at the intermediate regularized level. Explicit violations of Physical Unitarity for $m \neq 0$ can be studied e.g. by using the technique of 45.

The classical linearized ST operator [7] is

$$
\begin{aligned}
\mathcal{S}_{0}=\int d^{4} x & \left(\left(D_{\mu} \omega\right)^{a} \frac{\delta}{\delta A_{\mu}^{a}}-\frac{1}{2} f^{a b c} \omega^{b} \omega^{c} \frac{\delta}{\delta \omega^{a}}+B^{a} \frac{\delta}{\delta \bar{\omega}^{a}}\right. \\
& +i \omega^{a} T^{a} \psi_{i} \frac{\delta}{\delta \psi_{i}}+i \bar{\psi}_{i} T^{a} \omega^{a} \frac{\delta}{\delta \bar{\psi}_{i}} \\
& +\frac{\delta \Gamma_{m}^{(0)}}{\delta A^{a \mu}} \frac{\delta}{\delta A_{\mu}^{a *}}+\frac{\delta \Gamma_{m}^{(0)}}{\delta \omega^{a}} \frac{\delta}{\delta \omega^{a *}} \\
& \left.+\frac{\delta \Gamma_{m}^{(0)}}{\delta \psi_{i}} \frac{\delta}{\delta \bar{Y}_{i}}-\frac{\delta \Gamma_{m}^{(0)}}{\delta \bar{\psi}_{i}} \frac{\delta}{\delta Y_{i}}\right)+m^{2} \frac{\partial}{\partial \bar{\rho}}
\end{aligned}
$$

$\mathcal{S}_{0}$ is nilpotent as a consequence of eq. (15). 


\subsection{Additional symmetries of the model}

The dependence of the classical action $\Gamma_{m}^{(0)}$ on the Nakanishi-Lautrup multiplier field $B^{a}$ and on the antighost $\bar{\omega}^{a}$ is controlled by the following set of identities:

- the B-equation:

$$
\frac{\delta \Gamma_{m}^{(0)}}{\delta B^{a}}=\alpha B_{a}-\partial A_{a}-\bar{\rho} \omega_{a}
$$

- the antighost equation:

$$
\frac{\delta \Gamma_{m}^{(0)}}{\delta \bar{\omega}_{a}}=\partial_{\mu} \frac{\delta \Gamma_{m}^{(0)}}{\delta A_{\mu}^{a *}}-\bar{\rho} \frac{\delta \Gamma_{m}^{(0)}}{\delta \omega^{a *}}+m^{2} \omega^{a} .
$$

Both relations can be translated at the quantum level. Therefore the symmetric quantum vertex functional $\mathbb{\Gamma}_{m}$ satisfies

$$
\begin{aligned}
& \frac{\delta \Pi_{m}}{\delta B^{a}}=\alpha B_{a}-\partial A_{a}-\bar{\rho} \omega_{a}, \\
& \frac{\delta \Pi_{m}}{\delta \bar{\omega}_{a}}=\partial_{\mu} \frac{\delta \Pi_{m}}{\delta A_{\mu}^{a *}}-\bar{\rho} \frac{\delta \Pi_{m}}{\delta \omega^{a *}}+m^{2} \omega^{a} .
\end{aligned}
$$

We expand $\mathbb{\Gamma}_{m}$ in powers of $\hbar$ as follows:

$$
\mathbb{\Gamma}_{m}=\sum_{n=0}^{\infty} \mathbb{\Gamma}_{m}^{(j)}
$$

where $\mathbb{\Gamma}_{m}^{(j)}$ denotes the coefficient of $\mathbb{\Gamma}_{m}$ of order $j$ in the loop expansion. $\mathbb{\Gamma}_{m}^{(0)}$ coincides with $\Gamma_{m}^{(0)}$. By projecting eq. (19) at order $j \geq 1$ it follows that $\Gamma_{m}^{(j)}$ is $B^{a}$-independent. Moreover, by projection of eq. (20) at order $j \geq 1 \Gamma_{m}^{(j)}$ is seen to depend on $\bar{\omega}_{a}$ only via the combinations

$$
A_{\mu}^{a *^{\prime}}=A_{\mu}^{a *}-\partial_{\mu} \bar{\omega}^{a}, \quad \omega^{a *^{\prime}}=\omega^{a *}+\bar{\rho} \bar{\omega}^{a} .
$$

Their variations under $\mathcal{S}_{0}$ are

$$
\begin{aligned}
& \mathcal{S}_{0}\left(A_{\mu}^{a *^{\prime}}\right)=\left.\frac{\delta \Gamma_{m}^{(0)}}{\delta A_{\mu}^{a}}\right|_{B^{a}=0}, \\
& \mathcal{S}_{0}\left(\omega^{a *^{\prime}}\right)=\left.\frac{\delta \Gamma_{m}^{(0)}}{\delta \omega^{a}}\right|_{B^{a}=0}+m^{2} \bar{\omega}^{a}=\left.\frac{\delta \Gamma_{m}^{(0)}}{\delta \omega^{a}}\right|_{B^{a}=0, m^{2}=0} .
\end{aligned}
$$

\section{One-loop ST identities}

At one loop level the ST identities are

$$
\mathcal{S}_{0}\left(\mathbb{\Gamma}_{m}^{(1)}\right)=0 .
$$


It is useful to isolate in $\mathcal{S}_{0}$ the term acting on $\bar{\rho}$. Thus we define the differential $\delta$ according to

$$
\mathcal{S}_{0}=\delta+m^{2} \frac{\partial}{\partial \bar{\rho}}
$$

Since the counting operator $\mathcal{N}=\bar{\rho} \frac{\partial}{\partial \bar{\rho}}+m^{2} \frac{\partial}{\partial m^{2}}$ does not commute with $\mathcal{S}_{0}$ $\left(\bar{\rho}, m^{2}\right)$ form a set of BRST coupled doublets [46]. As such they do not contribute to the cohomology of $\mathcal{S}_{0}$. In the present model it is possible to remove the dependence of $\delta$ on $\left(\bar{\rho}, m^{2}\right)$ by a suitable change of coordinates [47]- 48], [13]. The appropriate redefinition is given by

$$
\hat{A}_{\mu}^{a *}=A_{\mu}^{a *^{\prime}}-\bar{\rho} A_{\mu}^{a}, \quad \hat{\omega}^{a *}=\omega^{a *^{\prime}} .
$$

One finds

$$
\begin{aligned}
& \mathcal{S}_{0}\left(\hat{A}_{\mu}^{a *}\right)=\left.\frac{\delta \Gamma_{m}^{(0)}}{\delta A_{\mu}^{a}}\right|_{B^{a}=0, m^{2}=0}, \\
& \mathcal{S}_{0}\left(\hat{\omega}^{a *}\right)=\left.\frac{\delta \Gamma_{m}^{(0)}}{\delta \omega^{a}}\right|_{B^{a}=0, m^{2}=0},
\end{aligned}
$$

where the R.H.S. in the above equation is expressed in terms of $\hat{A}_{\mu}^{a *}, \hat{\omega}^{a *}$. We notice that the $\mathcal{S}_{0}$-variations of $\hat{A}_{\mu}^{a *}, \hat{\omega}^{a *}$ are in the new variables $\bar{\rho}$ - and $m^{2}$ independent. Therefore in the new variables $\delta$ commutes with both $\mathcal{N}_{\bar{\rho}}=\bar{\rho} \frac{\partial}{\partial \bar{\rho}}$ and $\mathcal{N}_{m^{2}}=m^{2} \frac{\partial}{\partial m^{2}}$. From the nilpotency of $\mathcal{S}_{0}$ and the fact that $\left\{m^{2} \frac{\partial}{\partial \bar{\rho}}, \delta\right\}=0$ it follows that $\delta$ is also nilpotent.

The existence of the set of coordinates in eq. (26) has to be traced back to the fact that $\left(\bar{\rho}, m^{2}\right)$ enter in $\Gamma_{m}^{(0)}$ in a cohomologically trivial way. From now on we will work with the hatted variables in eq.(26).

\subsection{Construction of the one-loop solution}

The presence of the IR regulator $m$ in eq. (111) allows to perform the expansion of the 1-PI Green functions around zero momentum. We can then define the projector $t^{4}$ on the action-like part of $\mathbb{\Gamma}_{m}^{(1)}$ as follows. Let $\Phi_{I}, I=1, \ldots, N$ stand for the fields, antifields and external sources of the model and let $d\left(\Phi_{I}\right)$ be the UV dimension of $\Phi_{I}$. For any $n$-tuple $\underline{j}=\left\{j_{1}, \ldots, j_{n}\right\}$ we set $d(\underline{j})=$ $\sum_{k=1}^{n} d\left(\Phi_{j_{k}}\right)$ and $|\underline{j}|=n$, the length of $\underline{j}$. Then

$$
\begin{aligned}
t^{4} \mathbb{\Gamma}_{m}^{(1)}= & \sum_{n=1}^{\infty} \sum_{\substack{|\underline{j}|=n, d \underline{j}) \leq 4}} \int d^{4} p_{1} \ldots d^{4} p_{n} \delta^{(4)}\left(p_{1}+\ldots+p_{n}\right) \\
& \times\left. t^{4-d(\underline{j})} \frac{\delta^{(n)} \Gamma_{m}^{(1)}}{\delta \Phi_{j_{n}}\left(p_{n}\right) \ldots \delta \Phi_{j_{1}}\left(p_{1}\right)}\right|_{\Phi=0} \Phi_{j_{n}}\left(p_{n}\right) \ldots \Phi_{j_{1}}\left(p_{1}\right)
\end{aligned}
$$


where $t^{4-d(\underline{j})}$ is the Taylor operator around $p_{i}=0$ up to dimension $4-d(\underline{j})$ in the independent momenta $p_{i}, i=1, \ldots, n-1$.

In the configuration space $t^{4} \mathbb{\Gamma}_{m}^{(1)}$ is the projection of $\mathbb{\Gamma}_{m}^{(1)}$ on the sector spanned by all Lorentz-invariant monomials of dimension $\leq 4$ in the fields, the antifields, the external sources and their derivatives, compatible with the unbroken symmetries of the regularized action.

By the Quantum Action Principle [49]-[52] the one-loop BPHZL regularized, generally non-symmetric vertex functional $\Gamma_{m}^{(1)}$ may spoil the ST identities only by a local functional of dimension $\leq 5$ in the fields, the antifields, the external sources and their derivatives. Therefore in order to study the one-loop ST identites

$$
\mathcal{S}_{0}\left(\mathbb{\Gamma}_{m}^{(1)}\right)=0
$$

we can replace $\mathbb{\Gamma}_{m}^{(1)}$ with its effective part (i.e. the Taylor expansion of any amplitude in the independent external momenta around zero). This procedure associates to $\mathbb{\Gamma}_{m}^{(1)}$ a local formal power series given by an infinite sum of local Lorentz-invariant functionals. Without possibility of confusion we can denote this series by $\mathbb{\Gamma}_{m}^{(1)}$ itself.

Moreover, in the absence of anomalies this breaking can be compensated by adding to $\Gamma_{m}^{(1)}$ a local functional $\Upsilon^{(1)}$ in the fields, the antifields, the external sources and their derivatives of dimension $\leq 4$, in such a way that

$$
\mathbb{\Gamma}_{m}^{(1)}=\Gamma_{m}^{(1)}+\Upsilon^{(1)}, \quad t^{4} \Upsilon^{(1)}=\Upsilon^{(1)} .
$$

By the above equation it follows that

$$
\left(1-t^{4}\right) \Gamma_{m}^{(1)}=\left(1-t^{4}\right) \Gamma_{m}^{(1)} .
$$

Therefore the regularization procedure yields the correct symmetric Green functions in the whole sector of dimension $>4$. The only unknown part is the action-like part $t^{4} \Pi_{m}^{(1)}$. Once $\mathbb{\Gamma}_{m}^{(1)}$ is known $\Upsilon^{(1)}$ can be read off by comparison with eq.(30).

Eq.(29) defines a cohomological problem in the space of local formal power series spanned by $A_{\mu}^{a}, \bar{\psi}, \psi, \omega^{a}, \hat{\omega}^{a *}, \hat{A}_{\mu}^{a *}, Y_{i}, \bar{Y}_{i}, \bar{\rho}, m^{2}, \beta_{\mu}^{V}, \beta_{\mu}^{A}$ and their derivatives (without power-counting restrictions). Since we are interested only in $t^{4} \Gamma_{m}^{(1)}$ we rewrite eq.(29) as

$$
\mathcal{S}_{0}\left(t^{4} \Pi_{m}^{(1)}\right)=-\mathcal{S}_{0}\left(\left(1-t^{4}\right) \mathbb{\Gamma}_{m}^{(1)}\right) .
$$

It turns out [30] that an effective way to find the most general solution of eq. (32) is to parameterize the L.H.S. in terms of ST invariants (functionals belonging to the kernel of $\mathcal{S}_{0}$ ). On the other hand, the R.H.S. is expanded on a basis of monomials in the fields, the external sources and their derivatives. The coefficients of these monomials are given in terms of known superficially convergent vacuum amplitudes. Then eq.(32) is used to obtain directly the functional $t^{4} \mathbb{\Gamma}_{m}^{(1)}$. 
In order to apply this technique it is useful to decompose $\mathcal{S}_{0}$ according to the degree induced by the UV dimension. By inspection it is seen that $\delta$ splits into two components of degree zero and one respectively:

$$
\delta=\delta_{0}+\delta_{1},
$$

while $m^{2} \frac{\partial}{\partial \bar{\rho}}$ has degree one. $\delta_{0}$ keeps the dimension constant, while $\delta_{1}$ and $m^{2} \frac{\partial}{\partial \bar{\rho}}$ increase it by one. The action of $\delta_{0}$ and $\delta_{1}$ on the variables of the model is given in Appendix B

\subsection{ST parameterization of $t^{4} \mathbb{\Gamma}_{m}^{(1)}$}

By eq. (29) $\mathbb{\Gamma}_{m}^{(1)}$ belongs to the kernel of $\mathcal{S}_{0}$. The most general form of an arbitrary $\mathcal{S}_{0}$-invariant is known [53]-[55] and is given by

$$
\mathbb{\Gamma}_{m}^{(1)}=\sum_{j} \lambda_{j}^{(1)} \Lambda_{j}+\sum_{k} \rho_{k}^{(1)} \mathcal{S}_{0}\left(R_{k}\right)
$$

where $\Lambda_{j}$ are $\mathcal{S}_{0}$-invariants that are not $\mathcal{S}_{0}$-exact (i.e. they cannot be expressed as $\mathcal{S}_{0}$-variations of a functional $\Theta_{j}$ ) and $\mathcal{S}_{0}\left(R_{k}\right)$ are the trivial solutions (they are in the kernel of $\mathcal{S}_{0}$ since $\mathcal{S}_{0}$ is nilpotent).

For a semi-simple gauge group $G \Lambda_{j}$ are gauge-invariant polynomials built from the field strength $F_{\mu \nu}^{a}$ and its covariant derivatives, the matter fields and their covariant derivatives and the sources $\beta_{V}^{\mu}, \beta_{A}^{\mu}$, while all the dependence on the antifields and on $\bar{\rho}$ is confined to the trivial invariants.

If one wishes to parameterize the full $\mathbb{\Gamma}_{m}^{(1)}$ the whole infinite tower of functionals $\Lambda_{j}$ and $\mathcal{S}_{0}\left(R_{k}\right)$ is needed. However, since we are interested only in $t^{4} \mathbb{\Gamma}_{m}^{(1)}$, a finite number of them has to be considered. All functionals $\Lambda_{j}$ and $\mathcal{S}_{0}\left(R_{k}\right)$ such that $t^{4} \Lambda_{j}=t^{4} \mathcal{S}_{0}\left(R_{k}\right)=0$ can be dropped.

On the other hand the invariants contributing to the action-like sector split into two distinct subsets. The first one is generated by those invariants $X$ such that $t^{4} X \neq 0,\left(1-t^{4}\right) X=0$. The coefficients of these invariants are not constrained by the ST identities and have to be fixed by the choice of a complete set of normalization conditions. In addition there may also appear invariants such that $t^{4} X \neq 0,\left(1-t^{4}\right) X \neq 0$. Their coefficients are not free but fixed by superficially convergent Feynman amplitudes. They typically appear in the presence of cohomologically non-trivial mass parameters. In this model they must be of the trivial form (i.e. they are $\mathcal{S}_{0}$-exact) and fulfill $t^{4} \mathcal{S}_{0}\left(R_{k}\right) \neq$ $0,\left(1-t^{4}\right) \mathcal{S}_{0}\left(R_{k}\right) \neq 0$. They contain at least one $Y_{i}, \bar{Y}_{i}$ because the only component of $\mathcal{S}_{0}$ which does not increase the UV dimension is $\delta_{0}$ and the latter acts non-trivially only on the fermionic antifields $\bar{Y}_{i}, Y_{i}$.

The presence in the model of invariants of this kind is the reason why nontrivial non-symmetric terms, depending on superficially convergent amplitudes, have to be included in the action-like sector of $\mathbb{\Gamma}_{m}^{(1)}$ in order to fulfill the ST identities. 
Our next step is to find the complete set of invariants needed to parameterize $t^{4} \mathbb{\Gamma}_{m}^{(1)}$ (i.e. such that their action-like part is non-vanishing).

We first list those invariants $X$ such that their non-action-like part $\left(1-t^{4}\right) X$ vanishes. They can be classified according to the following groups:

- cohomologically non-trivial invariants independent of $\beta_{V}^{\mu}, \beta_{A}^{\mu}$ :

$$
\begin{aligned}
& \Lambda_{1}=\int d^{4} x F_{\mu \nu}^{a} F^{\mu \nu a}, \\
& \Lambda_{2}=\int d^{4} x \bar{\psi}_{i} \psi_{i} .
\end{aligned}
$$

We notice that the covariant kinetic term for the fermions,

$$
\int d^{4} x i \bar{\psi}_{i} \not D \psi_{i}
$$

is an independent BRST-invariant but not an independent $\mathcal{S}_{0}$-invariant (see eq.(39) and comments thereafter).

- cohomologically non-trivial invariants depending on $\beta_{V}^{\mu}, \beta_{A}^{\mu}$ :

$$
\begin{aligned}
& \Lambda_{V}=\int d^{4} x \beta_{\mu}^{V} \bar{\psi} \gamma^{\mu} \psi, \\
& \Lambda_{A}=\int d^{4} x \beta_{\mu}^{A} \bar{\psi} \gamma^{\mu} \gamma^{5} \psi,
\end{aligned}
$$

plus sixteen invariants only dependent on $\beta_{V}^{\mu}, \beta_{A}^{\mu}$ listed in Appendix $\mathbf{C}$

- cohomologically trivial invariants depending on $\bar{\rho}$

There is just one invariant of this kind:

$$
R_{\bar{\rho}}=\mathcal{S}_{0}\left(\int d^{4} x \frac{\bar{\rho}}{2}\left(A_{\mu}^{a}\right)^{2}\right)=\frac{m^{2}}{2} \int d^{4} x\left(A_{\mu}^{a}\right)^{2}-\int d^{4} x \bar{\rho} A_{\mu}^{a} \partial^{\mu} \omega^{a} .
$$

- cohomologically trivial invariants independent of $\bar{\rho}$ :

$$
\begin{aligned}
R_{1} & =\delta\left(\int d^{4} x \hat{A}_{\mu}^{a *} A_{a}^{\mu}\right) \\
R_{2} & =\delta\left(\int d^{4} x \hat{\omega}_{a}^{*} \omega_{a}\right) \\
R_{3} & =\frac{1}{2} \delta \int d^{4} x\left(\bar{\psi}_{i} Y_{i}-\bar{Y}_{i} \psi_{i}\right) \\
& =\int d^{4} x\left(i \bar{\psi}_{i} D \psi_{i}-M \bar{\psi}_{i} \psi_{i}+\beta_{\mu}^{V} \bar{\psi}_{i} \gamma^{\mu} \psi_{i}+\beta_{\mu}^{A} \bar{\psi}_{i} \gamma^{\mu} \gamma^{5} \psi_{i}\right)
\end{aligned}
$$

We notice that by the above equation the BRST-invariant

$$
\int d^{4} x i \bar{\psi}_{i} \not D \psi_{i}
$$


is in the same cohomology class of the sum

$$
\int d^{4} x\left(-M \bar{\psi}_{i} \psi_{i}+\beta_{\mu}^{V} \bar{\psi}_{i} \gamma^{\mu} \psi_{i}+\beta_{\mu}^{A} \bar{\psi}_{i} \gamma^{\mu} \gamma^{5} \psi_{i}\right)
$$

Therefore either $R_{3}$ or $\int d^{4} x i \bar{\psi}_{i} \not D \psi_{i}$ has to be included in the parameterization of $t^{4} \mathbb{\Gamma}_{m}^{(1)}$. In what follows we choose to use $\int d^{4} x i \bar{\psi}_{i} \not D \psi_{i}$ in order to achieve a direct control of the normalization condition for the residue of the fermionic field propagator.

The coefficients of the invariants in eqs. (35), (37), (38) and (39) are free and have to be fixed by providing a set of normalization conditions.

Let us consider now those invariants $X$ such that $t^{4} X \neq 0$ and $\left(1-t^{4}\right) X \neq 0$. They have to be $\mathcal{S}_{0}$-trivial and contain at least one $\bar{Y}_{i}, Y_{i}$. By power-counting they cannot depend on $\bar{\rho}$. The relevant invariants are

$$
\begin{aligned}
R_{4} & =\frac{1}{2} \delta\left(\int d^{4} x \bar{Y}_{i} A^{a} T^{a} \psi_{i}-\int d^{4} x \bar{\psi}_{i} T^{a} A^{a} Y_{i}\right), \\
R_{5} & =\frac{1}{2} \delta\left(\int d^{4} x i \bar{Y}_{i} \not \partial \psi_{i}-\int d^{4} x\left(-i \not \partial \bar{\psi}_{i} Y_{i}\right)\right), \\
R_{6} & =\delta \int d^{4} x i \bar{Y}_{i} T^{a} \omega^{a} Y_{i}, \\
R_{V} & =\delta \int d^{4} x\left(\beta_{\mu}^{V} \bar{Y}_{i} \gamma^{\mu} \psi_{i}-\beta_{\mu}^{V} \bar{\psi}_{i} \gamma^{\mu} Y_{i}\right), \\
R_{A} & =\delta \int d^{4} x\left(\beta_{\mu}^{A} \bar{Y}_{i} \gamma^{\mu} \gamma^{5} \psi_{i}-\beta_{\mu}^{A} \bar{\psi}_{i} \gamma^{\mu} \gamma^{5} Y_{i}\right) .
\end{aligned}
$$

Unlike the coefficients of the invariants in eqs. (35), (37)-(39) the coefficients of the invariants in eq. (40) are fixed by superficially convergent Feynman amplitudes.

By making use of these ST invariants the functional $t^{4} \Gamma_{m}^{(1)}$ solving eq. (32) can be written as

$$
\begin{aligned}
t^{4} \mathbb{\Gamma}^{(1)}= & \lambda_{1}^{(1)} \int d^{4} x F_{\mu \nu}^{a} F^{\mu \nu a}+\lambda_{2}^{(1)} \int d^{4} x \bar{\psi}_{i} \psi_{i}+\rho_{3}^{(1)} \int d^{4} x i \bar{\psi}_{i} \not \partial \psi_{i} \\
& +\sum_{i=1}^{16} \lambda_{\beta, i}^{(1)} \Lambda_{\beta, i}(x)+\lambda_{V}^{(1)} \int d^{4} x \beta_{\mu}^{V} \bar{\psi} \gamma^{\mu} \psi+\lambda_{A}^{(1)} \int d^{4} x \beta_{\mu}^{A} \bar{\psi} \gamma^{\mu} \gamma^{5} \psi \\
& +\rho_{\bar{\rho}}^{(1)}\left(\frac{m^{2}}{2} \int d^{4} x\left(A_{\mu}^{a}\right)^{2}-\int d^{4} x \bar{\rho} A_{\mu}^{a} \partial^{\mu} \omega^{a}\right) \\
& +\rho_{1}^{(1)} \delta \int d^{4} x\left(\hat{A}_{\mu}^{a *} A^{a \mu}\right)+\rho_{2}^{(1)} \delta \int d^{4} x\left(\hat{\omega}^{a *} \omega^{a}\right) \\
& +\frac{1}{2} \rho_{4}^{(1)} t^{4} \delta \int d^{4} x\left(\bar{Y}_{i} A^{a} T^{a} \psi_{i}-\bar{\psi}_{i} T^{a} A^{a} Y_{i}\right) \\
& +\frac{1}{2} \rho_{5}^{(1)} t^{4} \delta \int d^{4} x\left(i \bar{Y}_{i} \not \partial \psi_{i}+i \not \partial \bar{\psi}_{i} Y_{i}\right)
\end{aligned}
$$




$$
\begin{aligned}
& +\rho_{6}^{(1)} t^{4} \delta \int d^{4} x i \bar{Y}_{i} T^{a} \omega^{a} Y_{i} \\
& +\frac{\rho_{V}^{(1)}}{2} t^{4} \delta \int d^{4} x\left(\beta_{\mu}^{V} \bar{Y}_{i} \gamma^{\mu} \psi_{i}-\beta_{\mu}^{V} \bar{\psi}_{i} \gamma^{\mu} Y_{i}\right) \\
& +\frac{\rho_{A}^{(1)}}{2} t^{4} \delta \int d^{4} x\left(\beta_{\mu}^{A} \bar{Y}_{i} \gamma^{\mu} \gamma^{5} \psi_{i}-\beta_{\mu}^{A} \bar{\psi}_{i} \gamma^{\mu} \gamma^{5} Y_{i}\right) \\
& =\quad \lambda_{1}^{(1)} \int d^{4} x F_{\mu \nu}^{a} F^{\mu \nu a}+\lambda_{2}^{(1)} \int d^{4} x \bar{\psi}_{i} \psi_{i}+\rho_{3}^{(1)} \int d^{4} x i \bar{\psi}_{i} \not D \psi_{i}+\sum_{i=1}^{16} \lambda_{\beta, i}^{(1)} \Lambda_{\beta, i}(x) \\
& +\left(\lambda_{V}^{(1)}+M \rho_{V}^{(1)}\right) \int d^{4} x \beta_{\mu}^{V} \bar{\psi} \gamma^{\mu} \psi+\left(\lambda_{A}^{(1)}+M \rho_{A}^{(1)}\right) \int d^{4} x \beta_{\mu}^{A} \bar{\psi} \gamma^{\mu} \gamma^{5} \psi \\
& +\rho_{\bar{\rho}}^{(1)}\left(\frac{m^{2}}{2} \int d^{4} x\left(A_{\mu}^{a}\right)^{2}-\int d^{4} x \bar{\rho} A_{\mu}^{a} \partial^{\mu} \omega^{a}\right) \\
& +\rho_{1}^{(1)} \delta \int d^{4} x\left(\hat{A}_{\mu}^{a *} A^{a \mu}\right)+\rho_{2}^{(1)} \delta \int d^{4} x\left(\hat{\omega}^{a *} \omega^{a}\right) \\
& +\rho_{4}^{(1)} M \int d^{4} x \bar{\psi}_{i} A^{a} T^{a} \psi_{i}+\rho_{5}^{(1)} M \int d^{4} x i \bar{\psi}_{i} \not \partial \psi_{i} \\
& +\rho_{6}^{(1)} \int d^{4} x\left(i M \bar{\psi}_{i} T^{a} \omega^{a} Y_{i}-i M \bar{Y}_{i} T^{a} \omega^{a} \psi_{i}\right) .
\end{aligned}
$$

The dependence on $\rho_{V}^{(1)}, \rho_{A}^{(1)}$ can always be removed by performing the shifts

$$
\lambda_{V}^{(1)} \rightarrow \lambda_{V}^{(1)}+M \rho_{V}^{(1)}, \quad \lambda_{A}^{(1)} \rightarrow \lambda_{A}^{(1)}+M \rho_{A}^{(1)},
$$

which we assume from now on.

The free parameters of the solution are therefore $\lambda_{1}^{(1)}, \lambda_{2}^{(1)}, \rho_{3}^{(1)}, \lambda_{\beta, i}^{(1)}, \lambda_{V}^{(1)}, \lambda_{A}^{(1)}$ and $\rho_{\bar{\rho}}^{(1)}, \rho_{1}^{(1)}, \rho_{2}^{(1)}$. On the contrary $\rho_{4}^{(1)}, \rho_{5}^{(1)}$ and $\rho_{6}^{(1)}$ are fixed c-numbers (depending on superficially convergent Feynman amplitudes).

\subsection{Completing the construction}

In order to compute the fixed ST parameters $\rho_{4}^{(1)}, \rho_{5}^{(1)}$ and $\rho_{6}^{(1)}$ entering into eq.(41) we use eq.(32). We first compute the L.H.S. by applying $\mathcal{S}_{0}$ to eq.(41). This yields

$$
\begin{aligned}
\mathcal{S}_{0}\left(t^{4} \Gamma^{(1)}\right)= & M\left(\left(\rho_{5}^{(1)}-\rho_{4}^{(1)}\right)-\rho_{6}^{(1)}\right) \int d^{4} x \bar{\psi}_{i} \not \partial \omega^{a} T^{a} \psi_{i} \\
& -M \rho_{6}^{(1)} \int d^{4} x \bar{\psi}_{i} T^{a} f^{a b c} A^{b} \omega^{c} \psi_{i} \\
& -\frac{i M}{2} \rho_{6}^{(1)} \int d^{4} x\left(\bar{\psi}_{i} T^{a} f^{a b c} \omega^{b} \omega^{c} Y_{i}+\bar{Y}_{i} T^{a} f^{a b c} \omega^{b} \omega^{c} \psi_{i}\right) .
\end{aligned}
$$

On the other hand we expand $\left(1-t^{4}\right) \Gamma_{m}^{(1)}$ in a sum of Lorentz-invariant monomials of dimension $\geq 5$ in the fields, the antifields, the external sources and their 
derivatives. We only need to consider those monomials $\mathcal{N}_{j}$ whose $\mathcal{S}_{0}$-variation contains the monomials appearing in eq. (43). The relevant six $\mathcal{N}_{j}$ 's, whose coefficients we denote by $\gamma_{\mathcal{N}_{j}(x)}^{(1)}$, are listed in Appendix D By computing the $\mathcal{S}_{0}$-variation of $\left(1-t^{4}\right) \Pi_{m}^{(1)} \approx \sum_{j=1}^{6} \gamma_{\mathcal{N}_{j}(x)}^{(1)} \mathcal{N}_{j}$ we finally get

$$
\begin{aligned}
\mathcal{S}_{0}\left(\sum_{j=1}^{6} \gamma_{\mathcal{N}_{j}(x)}^{(1)} \mathcal{N}_{j}\right)= & i M\left(\gamma_{\mathcal{N}_{1}(x)}^{(1)}+\gamma_{\mathcal{N}_{2}(x)}^{(1)}-\gamma_{\mathcal{N}_{3}(x)}^{(1)}\right) \int d^{4} x \bar{\psi}_{i} \not \partial \omega^{a} T^{a} \psi_{i} \\
& +M\left(\gamma_{\mathcal{N}_{4}(x)}^{(1)}+\gamma_{\mathcal{N}_{5}(x)}^{(1)}\right) \int d^{4} x \bar{\psi}_{i} T^{a} f^{a b c} A^{b} \omega^{c} \psi_{i} \\
& +M \gamma_{\mathcal{N}_{6}(x)}^{(1)} \int d^{4} x i \bar{\psi}_{i} T^{a} f^{a b c} \omega^{b} \omega^{c} Y_{i} \\
& +M \gamma_{\mathcal{N}_{6}(x)}^{(1)} \int d^{4} x i \bar{Y}_{i} T^{a} f^{a b c} \omega^{b} \omega^{c} \psi_{i}
\end{aligned}
$$

By using eq.(43), eq.(44) and eq.(32) we obtain the following set of linear relations

$$
\begin{aligned}
& \rho_{6}^{(1)}=2 \gamma_{\mathcal{N}_{6}(x)}^{(1)}=\gamma_{\mathcal{N}_{4}(x)}^{(1)}+\gamma_{\mathcal{N}_{5}(x)}^{(1)}, \\
& \left(\rho_{5}^{(1)}-\rho_{4}^{(1)}\right)-\rho_{6}^{(1)}=-i\left(\gamma_{\mathcal{N}_{1}(x)}^{(1)}+\gamma_{\mathcal{N}_{2}(x)}^{(1)}-\gamma_{\mathcal{N}_{3}(x)}^{(1)}\right)
\end{aligned}
$$

Several comments are in order here. First we observe that the set of conditions in eqs. (45)-(46) allows to determine $\rho_{6}^{(1)}$ and the difference $\rho_{5}^{(1)}-\rho_{4}^{(1)}$ :

$$
\rho_{6}^{(1)}=2 \gamma_{\mathcal{N}_{6}(x)}^{(1)}, \quad \rho_{5}^{(1)}-\rho_{4}^{(1)}=2 \gamma_{\mathcal{N}_{6}(x)}^{(1)}-i\left(\gamma_{\mathcal{N}_{1}(x)}^{(1)}+\gamma_{\mathcal{N}_{2}(x)}^{(1)}-\gamma_{\mathcal{N}_{3}(x)}^{(1)}\right) .
$$

By exploiting the freedom in the choice of $\rho_{3}^{(1)}$ we redefine

$$
\rho_{3}^{(1)} \rightarrow \rho_{3}^{(1)}-M \rho_{5}^{(1)} .
$$

Then $t^{4} \Gamma_{m}^{(1)}$ in eq. (41) is seen to depend on $\rho_{4}^{(1)}, \rho_{5}^{(1)}$ only via the monomial

$$
\left(\rho_{3}^{(1)}-M\left(\rho_{5}^{(1)}-\rho_{4}^{(1)}\right)\right) \int d^{4} x \bar{\psi}_{i} A^{a} T^{a} \psi_{i}
$$

whose coefficient is controlled by the difference $\rho_{5}^{(1)}-\rho_{4}^{(1)}$. By using eqs. (47) we obtain finally the most general solution for the action-like part of the symmetric vertex functional $t^{4} \Gamma_{m}^{(1)}$ :

$$
\begin{aligned}
t^{4} \Gamma_{m}^{(1)}= & \lambda_{1}^{(1)} \int d^{4} x F_{\mu \nu}^{a} F^{\mu \nu a}+\lambda_{2}^{(1)} \int d^{4} x \bar{\psi}_{i} \psi_{i} \\
& +\sum_{i=1}^{16} \lambda_{\beta, i}^{(1)} \Lambda_{\beta, i}(x)+\lambda_{V}^{(1)} \int d^{4} x \beta_{\mu}^{V} \bar{\psi} \gamma^{\mu} \psi+\lambda_{A}^{(1)} \int d^{4} x \beta_{\mu}^{A} \bar{\psi} \gamma^{\mu} \gamma^{5} \psi \\
& +\rho_{\bar{\rho}}^{(1)}\left(\frac{m^{2}}{2} \int d^{4} x\left(A_{\mu}^{a}\right)^{2}-\int d^{4} x \bar{\rho} A_{\mu}^{a} \partial^{\mu} \omega^{a}\right)
\end{aligned}
$$




$$
\begin{aligned}
& +\rho_{1}^{(1)} \delta \int d^{4} x\left(\hat{A}_{\mu}^{a *} A^{a \mu}\right)+\rho_{2}^{(1)} \delta \int d^{4} x\left(\hat{\omega}^{a *} \omega^{a}\right) \\
& +\rho_{3}^{(1)} \int d^{4} x i \bar{\psi}_{i} \not \partial \psi_{i} \\
& +\left(\rho_{3}^{(1)}+i M\left(\gamma_{\mathcal{N}_{1}(x)}^{(1)}+\gamma_{\mathcal{N}_{2}(x)}^{(1)}-\gamma_{\mathcal{N}_{3}(x)}^{(1)}\right)-2 M \gamma_{\mathcal{N}_{6}(x)}^{(1)}\right) \int d^{4} x \bar{\psi}_{i} A^{a} T^{a} \psi_{i} \\
& +2 \gamma_{\mathcal{N}_{6}}^{(1)} \int d^{4} x\left(i M \bar{\psi}_{i} T^{a} \omega^{a} Y_{i}-i M \bar{Y}_{i} T^{a} \omega^{a} \psi_{i}\right) .
\end{aligned}
$$

The parameters $\lambda_{j}^{(1)}, j=1,2, \lambda_{\beta, i}^{(1)}, i=1, \ldots, 16, \lambda_{V}^{(1)}, \lambda_{A}^{(1)}, \rho_{\bar{\rho}}^{(1)}, \rho_{k}^{(1)}, k=$ $1,2,3$ are free parameters to be fixed by a suitable choice of normalization conditions. The coefficients $\gamma_{\mathcal{N}_{j}(x)}^{(1)}, j=1, \ldots, 6$ are given by vacuum diagrams associated to superficially convergent Feynman amplitudes (and their derivatives w.r.t external momenta) evaluated at zero momentum.

For $M=0$ the functional $t^{4} \mathbb{\Gamma}_{m}^{(1)}$ becomes $\mathcal{S}_{0}$-invariant. For $M \neq 0$ it is not $\mathcal{S}_{0}$-invariant since it contains a non-symmetric contribution to the gauge boson-fermion-fermion vertex and to the fermionic antifield couplings. These non-symmetric terms are required in order to fulfill the one-loop ST identities in eq. (24) for the complete functional $\mathbb{\Gamma}_{m}^{(1)}$. They are generated by the presence of cohomologically non-trivial mass parameters in the model.

We notice that with the choice $\rho_{\bar{\rho}}^{(1)}=0$ all terms entering in eq. (50) are IR safe, fulfilling the power-counting criteria of 38- 43 . Therefore IR convergence in the massless limit $m \rightarrow 0$ is guaranteed provided that the dependence of $\mathbb{\Gamma}_{m}^{(1)}$ on $\bar{\rho}$ only happens via the antifields in eq. (26).

\section{Consistency conditions}

We look back at eqs. (45) and (46). By eliminating the $\rho$ 's between the equations we find a set of consistency conditions (i.e. relations between superficially comvergent Feynman amplitudes that must be automatically fulfilled in any regularization scheme compatible with the Quantum Action Principle):

$$
2 \gamma_{\mathcal{N}_{6}(x)}^{(1)}=\gamma_{\mathcal{N}_{4}(x)}^{(1)}+\gamma_{\mathcal{N}_{5}(x)}^{(1)} .
$$

The origin of these consisitency conditions is the Wess-Zumino consistency condition valid for the ST breaking $\Delta^{(1)}=\mathcal{S}_{0}\left(\Gamma_{m}^{(1)}\right)$ :

$$
\mathcal{S}_{0}\left(\Delta^{(1)}\right)=0 .
$$

$\Gamma_{m}^{(1)}$ is any generally non-symmetric vertex functional obtained by a regularization procedure consistent with the Quantum Action Principle and including a generic set of finite counterterms compatible with power-counting. As is clear, eq. (52) is a direct consequence of the nilpotency of $\mathcal{S}_{0}$. 
If one does not impose eq. (47) but keep $\rho_{4}^{(1)}, \rho_{5}^{(1)}, \rho_{6}^{(1)}$ arbitrary the ST identities are broken. The breaking term is given by

$$
\begin{aligned}
\Delta^{(1)}= & \int d^{4} x\left(a^{(1)} \bar{\psi}_{i} \not \omega^{a} T^{a} \psi_{i}+b^{(1)} \bar{\psi}_{i} T^{a} f^{a b c} \not^{b} \omega^{c} \psi_{i}\right. \\
& \left.+e^{(1)} i \bar{\psi}_{i} T^{a} f^{a b c} \omega^{b} \omega^{c} Y_{i}+\bar{e}^{(1)} i \bar{Y}_{i} T^{a} f^{a b c} \omega^{b} \omega^{c} \psi_{i}\right) .
\end{aligned}
$$

The explicit values of the coefficients $a^{(1)}, b^{(1)}, e^{(1)}$ and $\bar{e}^{(1)}$ are

$$
\begin{aligned}
a^{(1)} & =M\left[\left(\rho_{5}^{(1)}-\rho_{4}^{(1)}\right)-\rho_{6}^{(1)}+i\left(\gamma_{\mathcal{N}_{1}(x)}^{(1)}+\gamma_{\mathcal{N}_{2}(x)}^{(1)}-\gamma_{\mathcal{N}_{3}(x)}^{(1)}\right)\right], \\
b^{(1)} & =-M \rho_{6}^{(1)}+M \gamma_{\mathcal{N}_{5}(x)}^{(1)}+M \gamma_{\mathcal{N}_{6}(x)}^{(1)}, \\
e^{(1)} & =-\frac{M}{2} \rho_{6}^{(1)}+M \gamma_{\mathcal{N}_{6}(x)}^{(1)}, \\
\bar{e}^{(1)} & =-\frac{M}{2} \rho_{6}^{(1)}+M \gamma_{\mathcal{N}_{6}(x)}^{(1)} .
\end{aligned}
$$

We can now compute the $\mathcal{S}_{0}$-variation of $\Delta^{(1)}$ :

$$
\begin{array}{rl}
\mathcal{S}_{0}\left(\Delta^{(1)}\right)=\int d^{4} & x \\
& \left(\left(e^{(1)}-\bar{e}^{(1)}\right)\left(-i M \bar{\psi}_{i} T^{a} f^{a b c} \omega^{b} \omega^{c} \psi_{i}\right)\right. \\
& +\left(e^{(1)}-\bar{e}^{(1)}\right)\left(-\bar{\psi}_{i} T^{a} f^{a b c} \omega^{b} \omega^{c} \not \partial \psi_{i}\right) \\
& +e^{(1)}\left(i \bar{\psi}_{i} f^{a b c} \omega^{b} \omega^{c} T^{a} T^{d} A^{d} \psi_{i}\right)+\bar{e}^{(1)}\left(-i \bar{\psi}_{i} A^{d} T^{d} T^{a} f^{a b c} \omega^{b} \omega^{c} \psi_{i}\right) \\
& +\left(2 \bar{e}^{(1)}-b^{(1)}\right) \bar{\psi}_{i} f^{a b c} \not \partial \omega^{b} \omega^{c} T^{a} \psi_{i} \\
& \left.-b^{(1)} \bar{\psi}_{i} \frac{T^{a}}{2} f^{a d j} f^{j b c} A^{d} \omega^{b} \omega^{c} \psi_{i}\right)
\end{array}
$$

The above expression has to be zero according to eq.(52). The first and second line then yield

$$
e^{(1)}=\bar{e}^{(1)} .
$$

The fourth line gives

$$
b^{(1)}=2 \bar{e}^{(1)} .
$$

By using eq.(56) into eq.(55) eq.(155) becomes after some algebra

$$
\begin{aligned}
\mathcal{S}_{0}\left(\Delta^{(1)}\right)=\int d^{4} x & \left(\left(2 \bar{e}^{(1)}-b^{(1)}\right) \bar{\psi}_{i} f^{a b c} \not \partial \omega^{b} \omega^{c} T^{a} \psi_{i}\right. \\
+ & \left.\left(e^{(1)}-\frac{b^{(1)}}{2}\right) \bar{\psi}_{i} \frac{T^{a}}{2} f^{a d j} f^{j b c} \not A^{d} \omega^{b} \omega^{c} \psi_{i}\right) .
\end{aligned}
$$

This yields in addition to eqs. (156)-(157)

$$
e^{(1)}=\frac{b^{(1)}}{2}
$$


which is indeed a consequence of eqs.(56)-(57). The condition in eq. (56) guarantees the equality of the coefficients of the monomials $\int d^{4} x i \bar{\psi}_{i} T^{a} f^{a b c} \omega^{b} \omega^{c} Y_{i}$ and its bar-conjugated $\int d^{4} x i \bar{Y}_{i} T^{a} f^{a b c} \omega^{b} \omega^{c} \psi_{i}$, which is verified by the expressions in eq.(54). On the other hand eq.(57) gives

$$
\begin{aligned}
& 0=-M \rho_{6}^{(1)}+M \gamma_{\mathcal{N}_{4}(x)}^{(1)}+M \gamma_{\mathcal{N}_{5}(x)}^{(1)}-2\left(-\frac{M}{2} \rho_{6}^{(1)}+M \gamma_{\mathcal{N}_{6}(x)}^{(1)}\right) \\
& =M \gamma_{\mathcal{N}_{4}(x)}^{(1)}+M \gamma_{\mathcal{N}_{5}(x)}^{(1)}-2 M \gamma_{\mathcal{N}_{6}(x)}^{(1)},
\end{aligned}
$$

i.e. the consistency condition in eq.(51).

\section{$5 \quad$ Higher orders}

Higher order ST identities involve the solution of an inhomogeneous equation for $t^{4} \Gamma_{m}^{(n)}, n>1$ :

$$
\mathcal{S}_{0}\left(t^{4} \mathbb{\Gamma}_{m}^{(n)}\right)=-\mathcal{S}_{0}\left(\left(1-t^{4}\right) \mathbb{\Gamma}_{m}^{(n)}\right)-\sum_{j=1}^{n-1}\left(\mathbb{\Gamma}^{(j)}, \mathbb{\Gamma}^{(n-j)}\right) .
$$

The parenthesis in the above equation is defined by

$$
(X, Y)=\int d^{4} x\left(\frac{\delta X}{\delta \hat{A}_{\mu}^{a *}} \frac{\delta Y}{\delta A^{a \mu}}+\frac{\delta X}{\delta \hat{\omega}^{a *}} \frac{\delta Y}{\delta \omega^{a}}-\frac{\delta X}{\delta Y_{i}} \frac{\delta Y}{\delta \bar{\psi}_{i}}+\frac{\delta X}{\delta \bar{Y}_{i}} \frac{\delta Y}{\delta \psi_{i}}\right) .
$$

The R.H.S. of the eq. 61) is known (it contains superficially convergent $n$-order Feynman amplitudes and known lower dimensional contributions coming from the bracket $\left(\Gamma^{(j)}, \Gamma^{(n-j)}\right)$ ). Again we can limit ourselves to the projection of eq. (61) on the subspace of dimension $\leq 5$ in the fields, antifields, external sources and their derivatives of ghost number one, since by the QAP higher dimensional terms are absent (under the recursive assumption that the ST identities have been restored up to order $n-1)$.

Since $\mathcal{S}_{0}$ is nilpotent the $\mathcal{S}_{0}$-variation of eq.61) gives

$$
\mathcal{S}_{0}\left(\sum_{j=1}^{n-1}\left(\Pi^{(j)}, \Pi^{(n-j)}\right)\right)=0 .
$$

In the absence of anomalies by eq. (63) there exists a functional $\Theta^{(n)}$ such that

$$
\sum_{j=1}^{n-1}\left(\Gamma^{(j)}, \Pi^{(n-j)}\right)=\mathcal{S}_{0}\left(\Theta^{(n)}\right) .
$$

This functional is not unique (one can add any $\mathcal{S}_{0}$-invariant with ghost number zero to $\Theta^{(n)}$ without violating eq.(64)). The knowledge of any particular solution to eq. (64) is enough for the present purposes. Since the L.H.S. of eq. (64) is 
completely fixed in terms of lower order Feynman amplitudes, the determination of $\Theta^{(n)}$ is a purely algebraic problem. $\Theta^{(n)}$ :

We insert eq. 64) in eq. (61) and obtain a homogeneous equation for $\mathbb{\Gamma}_{m}^{(n)}+$

$$
\mathcal{S}_{0}\left(\mathbb{\Gamma}_{m}^{(n)}+\Theta^{(n)}\right)=0 .
$$

At this stage the technique developed in Sect. 3 3 can be applied. We obtain

$$
\mathcal{S}_{0}\left(t^{4} \mathbb{\Gamma}_{m}^{(n)}\right)=-\mathcal{S}_{0}\left(\left(1-t^{4}\right) \mathbb{\Gamma}_{m}^{(n)}\right)-\mathcal{S}_{0}\left(\Theta^{(n)}\right) .
$$

The R.H.S. is known. The contribution from the bracket in eq. 61), which controls the non-linearity of higher-order ST identities, is taken into account by the last term in the R.H.S. of eq. (66).

The explicit determination of the functional $\Theta^{(n)}$ for arbitrary values of the free parameters $\lambda^{(j)}, \rho^{(j)}, j<n$ may be a difficult task and results in very complicated expressions. Examples for the pure Yang-Mills sector are given in [30.

However the problem simplifies considerably if the freedom in the choice of $\lambda^{(j)}, \rho^{(j)}$ is used in order to achieve the simplest possible form of the contribution generated by the bracket in eq. (61).

Let us illustrate this point on the model at hand. We start from two-loop order. It turns out that in the present model there is a natural choice of the normalization conditions such that

$$
\left(\mathbb{\Gamma}^{(1)}, \mathbb{\Gamma}^{(1)}\right)=0 \quad \text { up to dimension } 5 .
$$

The proof is as follows. Let us choose

$$
\lambda_{1}^{(1)}=0, \quad \lambda_{2}^{(1)}=0, \quad \rho_{1}^{(1)}=0, \quad \rho_{\bar{\rho}}^{(1)}=0 .
$$

Moreover we choose $\rho_{2}^{(1)}$ in order to cancel the $\bar{Y}_{i}, Y_{i}$-terms in $t^{4} \mathbb{\Gamma}^{(1)}$. This can be done since

$$
\begin{aligned}
& \mathcal{S}_{0}\left(\int d^{4} x \hat{\omega}^{a *} \omega^{a}\right)= \delta\left(\int d^{4} x \hat{\omega}^{a *} \omega^{a}\right) \\
&=\int d^{4} x\left(-\omega^{a} \frac{\delta \Gamma^{(0)}}{\delta \omega^{a}}+\hat{\omega}^{a *}\left(-\frac{1}{2} f^{a b c} \omega^{b} \omega^{c}\right)\right) \\
&=\int d^{4} x\left(-\hat{A}_{\mu}^{* a}\left(D^{\mu} \omega\right)^{a}-\hat{\omega}^{a *}\left(-\frac{1}{2} f^{a b c} \omega^{b} \omega^{c}\right)\right. \\
&\left.\quad+i \bar{\psi}_{i} T^{a} \omega^{a} Y_{i}-i \bar{Y}_{i} \omega^{a} T^{a} \psi_{i}\right) .
\end{aligned}
$$

By choosing

$$
\rho_{2}^{(1)}=-2 M \gamma_{\mathcal{N}_{6}}^{(1)}
$$

we obtain the desired condition on $t^{4} \Gamma^{(1)}$. 
Finally we choose $\rho_{3}^{(1)}$ in such a way to set the coefficient of the monomial $\int d^{4} x \bar{\psi}_{i} A^{a} T^{a} \psi_{i}$ equal to zero, namely

$$
\rho_{3}^{(1)}=-i M\left(\gamma_{\mathcal{N}_{1}(x)}^{(1)}+\gamma_{\mathcal{N}_{2}(x)}^{(1)}-\gamma_{\mathcal{N}_{3}(x)}^{(1)}\right)+2 M \gamma_{\mathcal{N}_{6}(x)}^{(1)} .
$$

The resulting expression for $t^{4} \mathbb{\Gamma}^{(1)}$ is

$$
\begin{aligned}
t^{4} \mathbb{\Gamma}^{(1)}= & +\sum_{i=1}^{16} \lambda_{\beta, i}^{(1)} \Lambda_{\beta, i}(x)+\lambda_{V}^{(1)} \int d^{4} x \beta_{\mu}^{V} \bar{\psi} \gamma^{\mu} \psi+\lambda_{A}^{(1)} \int d^{4} x \beta_{\mu}^{A} \bar{\psi} \gamma^{\mu} \gamma^{5} \psi \\
& +\left.\rho_{2}^{(1)} \delta \int d^{4} x\left(\hat{\omega}^{a *} \omega^{a}\right)\right|_{Y_{i}=\bar{Y}_{i}=0}+\rho_{3}^{(1)} \int d^{4} x i \bar{\psi}_{i} \not \partial \psi_{i}
\end{aligned}
$$

By eq.(72)

$\left(t^{4} \Pi^{(1)}, t^{4} \Pi^{(1)}\right)=\left(\rho_{2}^{(1)}\right)^{2}\left(\left.\delta \int d^{4} x\left(\hat{\omega}^{a *} \omega^{a}\right)\right|_{Y_{i}=\bar{Y}_{i}=0},\left.\delta \int d^{4} x\left(\hat{\omega}^{a *} \omega^{a}\right)\right|_{Y_{i}=\bar{Y}_{i}=0}\right)$.

By power-counting, since $\lambda_{2}^{(1)}$ has been chosen equal to zero, $\left(t^{4} \Gamma^{(1)}, t^{4} \Pi^{(1)}\right)$ coincides up to dimension 5 with $\left(\mathbb{\Gamma}^{(1)}, \mathbb{\Gamma}^{(1)}\right)$. Therefore we can limit ourselves to the study of eq.(73).

The R.H.S. of eq.(73) is zero. This can be easily seen by noticing that from eq. (69) $\left.\delta \int d^{4} x\left(\hat{\omega}^{a *} \omega^{a}\right)\right|_{Y_{i}=\bar{Y}_{i}=0}$ is, up to an overall minus sign, the antifielddependent part of the classical action of pure Yang-Mills theory. Eq. (173) is nothing but the classical ST identities for $\left.\delta \int d^{4} x\left(\hat{\omega}^{a *} \omega^{a}\right)\right|_{Y_{i}=\bar{Y}_{i}=0}$. Since the latter is BRST invariant, eq. (173) vanishes. Therefore there exists a choice of normalization conditions given by eqs. (68), (70) and (71) allowing to discard the inhomogeneous contribution originated by the bracket $\left(\mathbb{\Gamma}^{(1)}, \mathbb{\Gamma}^{(1)}\right)$ in the relevant sector of dimension $\leq 5$. With this special choice of normalization conditions the two-loop ST identities read

$$
\mathcal{S}_{0}\left(\mathbb{\Gamma}^{(2)}\right)=0 .
$$

The discussion of eq. (74) proceeds in the same way as for eq.(24). The argument can be recursively repeated to all orders in the loop expansion. If the following normalization conditions are imposed order by order in perturbation theory for $j=1,2, \ldots, n-1$

$$
\begin{aligned}
& \lambda_{1}^{(j)}=0, \quad \lambda_{2}^{(j)}=0, \quad \rho_{1}^{(j)}=0, \quad \rho_{\bar{\rho}}^{(j)}=0, \\
& \rho_{2}^{(j)}=-2 M \gamma_{\mathcal{N}_{6}}^{(j)}, \quad \rho_{3}^{(j)}=-i M\left(\gamma_{\mathcal{N}_{1}(x)}^{(j)}+\gamma_{\mathcal{N}_{2}(x)}^{(j)}-\gamma_{\mathcal{N}_{3}(x)}^{(j)}\right)+2 M \gamma_{\mathcal{N}_{6}(x)}^{(j)}
\end{aligned}
$$

the action-like part of the $n$-th order symmetric vertex functional is

$$
t^{4} \Gamma^{(n)}=\lambda_{1}^{(n)} \int d^{4} x F_{\mu \nu}^{a} F^{\mu \nu a}+\lambda_{2}^{(n)} \int d^{4} x \bar{\psi}_{i} \psi_{i}
$$




$$
\begin{aligned}
& +\sum_{i=1}^{16} \lambda_{\beta, i}^{(n)} \Lambda_{\beta, i}(x)+\lambda_{V}^{(n)} \int d^{4} x \beta_{\mu}^{V} \bar{\psi} \gamma^{\mu} \psi+\lambda_{A}^{(n)} \int d^{4} x \beta_{\mu}^{A} \bar{\psi} \gamma^{\mu} \gamma^{5} \psi \\
& +\rho_{\bar{\rho}}^{(n)}\left(\frac{m^{2}}{2} \int d^{4} x\left(A_{\mu}^{a}\right)^{2}-\int d^{4} x \bar{\rho} A_{\mu}^{a} \partial^{\mu} \omega^{a}\right) \\
& +\rho_{1}^{(n)} \delta \int d^{4} x\left(\hat{A}_{\mu}^{a *} A^{a \mu}\right)+\rho_{2}^{(n)} \delta \int d^{4} x\left(\hat{\omega}^{a *} \omega^{a}\right) \\
& +\rho_{3}^{(n)} \int d^{4} x i \bar{\psi}_{i} \not \partial \psi_{i} \\
& +\left(\rho_{3}^{(n)}+i M\left(\gamma_{\mathcal{N}_{1}(x)}^{(n)}+\gamma_{\mathcal{N}_{2}(x)}^{(n)}-\gamma_{\mathcal{N}_{3}(x)}^{(n)}\right)-2 M \gamma_{\mathcal{N}_{6}(x)}^{(n)}\right) \int d^{4} x \bar{\psi}_{i} A^{a} T^{a} \psi_{i} \\
& +2 \gamma_{\mathcal{N}_{6}}^{(n)} \int d^{4} x\left(i M \bar{\psi}_{i} T^{a} \omega^{a} Y_{i}-i M \bar{Y}_{i} T^{a} \omega^{a} \psi_{i}\right) .
\end{aligned}
$$

Moreover, with the choice $\rho_{\bar{\rho}}^{(j)}=0, j=1, \ldots, n$ all terms entering in eq.(50) are IR safe, fulfilling the power-counting criteria of 38- 43. This in turn implies that the massless limit $m \rightarrow 0(s \rightarrow 1)$ of $\mathbb{\Gamma}_{m}$ exist:

$$
\mathbb{\Gamma}=\lim _{m \rightarrow 0} \mathbb{\Gamma}_{m}
$$

and obeys the following ST identities of Yang-Mills theory with massive fermions

$$
\begin{aligned}
& \mathcal{S}(\mathbb{\Gamma})=\int d^{4} x\left(\frac{\delta \mathbb{\Gamma}}{\delta A_{\mu}^{a *}} \frac{\delta \mathbb{\Gamma}}{\delta A^{a \mu}}+\frac{\delta \mathbb{\Gamma}}{\delta \omega^{a *}} \frac{\delta \mathbb{\Gamma}}{\delta \omega^{a}}+B^{a} \frac{\delta \mathbb{\Gamma}}{\delta \bar{\omega}^{a}}\right. \\
& \left.-\frac{\delta \Pi}{\delta Y_{i}} \frac{\delta \Pi}{\delta \bar{\psi}_{i}}+\frac{\delta \Pi}{\delta \bar{Y}_{i}} \frac{\delta \Pi}{\delta \psi_{i}}\right)=0 .
\end{aligned}
$$

\section{Conclusions}

By applying the method of the ST parameterization of the symmetric (fulfilling the ST identities) vertex functional, a complete characterization of the action-like part of the symmetric quantum effective action has been achieved for Yang-Mills theory with massive fermions in the presence of singlet axial-vector currents.

An IR regulator $m$ has been introduced within the BPHZL scheme and the relevant extended ST identities, parameterizing the soft-breaking induced by the intermediate gauge bosons and ghost masses, have been analyzed. The IR regulated symmetric vertex functional $\mathbb{\Gamma}_{m}$ has been constructed. The limit $\mathbb{\Gamma}_{m}$ for $m \rightarrow 0$ is seen to exist since the BPHZL IR power-counting criteria hold true at any order in the loop expansion. In this limit the extended ST identities reduce to those of Yang-Mills theory with massive fermions in eq. (78).

The ST parameterization reveals an interesting geometric structure also for the finite action-like part of the symmetric quantum effective action.

Since a cohomologically non-trivial mass parameter like the fermion mass $M$ enters into the theory, the action-like functional $t^{4} \mathbb{\Gamma}_{m}$ is not invariant under 
the linearized classical ST operator $\mathcal{S}_{0}$. The non-invariant action-like terms proportional to $M$ are needed in order to fulfill the ST identities for the whole vertex functional $\mathbb{\Gamma}_{m}$.

Moreover, we have shown that a natural set of normalization conditions exists allowing to reduce higher-order ST identities to a linear homogeneous problem. This is a rather non-trivial consequence of the geometric structure of $t^{4} \mathbb{\Gamma}_{m}$ unveiled by the ST parameterization of the quantum effective action.

By exploiting this set of normalization conditions the expression of the action-like part of the symmetric vertex functional to any order in the loop expansion has been given.

We remark that the inclusion of composite operators like the singlet axialvector currents does not pose any additional problem in the application of the ST parameterization technique. Hence the latter also provides an efficient way to treat BRST-invariant composite operators in those theories where no invariant regularization scheme is known.

We would like finally to comment on the use of the BPHZL regularization scheme in order to construct the symmetric $n$-th order quantum effective action. In this scheme an IR regulator is introduced in a way consistent with powercounting (so that each factor of $m=\mu(s-1)$ counts as one).

It should be noticed that the algebraic structure underlying the use of the IR regulator is independent of the particular regularization scheme adopted and can be thus translated without difficulty to other regularization procedures, like for instance Dimensional Regularization (DR) (in the formulation compatible with the Quantum Action Principle of [49, 56, 57). However, special care has to be paid to the subtraction chosen to deal with the IR regulator in order to ensure that the power-counting properties true for the BPHZL IR regulator are still valid. Provided that this requirement is fulfilled, the formulas given in eqs.(50) and (76) can be regarded as regularization scheme-independent results.

\section{Acknowledgments}

Useful discussions with R. Ferrari and D. Maison are gratefully acknowledged. The Author also would like to thank the warm hospitality of the Theory Group at Max-Planck-Institut für Physik (Werner-Heisenberg-Institut), where part of this work was completed.

\section{A BRST symmetry}

The BRST symmetry of the model is

$$
\begin{aligned}
& s A_{\mu}^{a}=\left(D_{\mu} \omega\right)^{a} \equiv \partial_{\mu} \omega^{a}+f^{a b c} A_{\mu}^{b} \omega^{c}, \quad s \omega^{a}=-\frac{1}{2} f^{a b c} \omega^{b} \omega^{c}, \\
& s \psi_{i}=i \omega^{a} T^{a} \psi_{i}, \quad s \bar{\psi}_{i}=i \bar{\psi}_{i} T^{a} \omega^{a} .
\end{aligned}
$$


The following conjugation rules hold:

$$
s \bar{b}=\overline{s b}, \quad s \bar{f}=-\overline{s f},
$$

with $b$ boson and $f$ fermion.

The introduction of the BPHZL mass regulator $m$ in eq.(13) requires an additional BRST pair $\left(\bar{\rho}, m^{2}\right)$

$$
s \bar{\rho}=m^{2}, \quad s m^{2}=0,
$$

with $\bar{\rho}$ an anticommuting constant parameter.

The IR regularized BRST-invariant classical action is

$$
\begin{aligned}
\Gamma_{m}^{(0)}=\int & d^{4} x\left(-\frac{1}{4 g^{2}} F_{\mu \nu}^{a} F^{\mu \nu a}+i \bar{\psi}_{i} D \psi_{i}-M \bar{\psi}_{i} \psi_{i}+\beta_{\mu}^{V} \bar{\psi} \gamma^{\mu} \psi+\beta_{\mu}^{A} \bar{\psi} \gamma^{\mu} \gamma^{5} \psi\right. \\
& +\frac{1}{2} m^{2}\left(A_{\mu}^{a}\right)^{2}+m^{2} \bar{\omega}^{a} \omega^{a}-\bar{\rho} A_{\mu}^{a} \partial^{\mu} \omega^{a}-\bar{\rho} B^{a} \omega^{a}-\frac{1}{2} \bar{\rho} \bar{\omega}^{a} f^{a b c} \omega^{b} \omega^{c} \\
& +\alpha \frac{\left(B^{a}\right)^{2}}{2}-B^{a} \partial A^{a}+\bar{\omega}^{a} \partial^{\mu}\left(D_{\mu} \omega\right)^{a} \\
& \left.+A_{\mu}^{a *}\left(D^{\mu} \omega\right)^{a}+\omega^{a *}\left(-\frac{1}{2} f^{a b c} \omega^{b} \omega^{c}\right)-i \bar{\psi}_{i} T^{a} \omega^{a} Y_{i}+i \bar{Y}_{i} \omega^{a} T^{a} \psi_{i}\right)
\end{aligned}
$$

The UV dimension of the fields, antifields and external sources is assigned according to the following table:

\begin{tabular}{|c|c|c|c|c|c|c|c|c|c|c|c|c|}
\hline Field & $A_{\mu}^{a}$ & $\omega^{a}$ & $\bar{\omega}^{a}$ & $B^{a}$ & $\psi_{i}$ & $\psi_{i}$ & $A_{\mu}^{a *}$ & $\omega_{a}^{*}$ & $Y_{i}$ & $Y_{i}$ & $\beta_{\mu}^{V}$ & $\beta_{\mu}^{A}$ \\
\hline UV dim. & 1 & 1 & 1 & 2 & $3 / 2$ & $3 / 2$ & 2 & 2 & $3 / 2$ & $3 / 2$ & 1 & 1 \\
\hline
\end{tabular}

Table 1 - UV dimension of the fields, antifields and external sources

$\bar{\psi}, \psi$ have IR dimension two. The IR dimension of the other fields coincides with the UV dimension. Under parity $\beta_{\mu}^{V}$ is even and $\beta_{\mu}^{A}$ odd. 


\section{B Decomposition of $\delta$}

The decomposition of $\delta$ according to the UV dimension of the fields, external sources and their derivatives is

$$
\begin{array}{ll}
\delta_{0} A_{\mu}^{a}=0, & \delta_{1} A_{\mu}^{a}=\left(D_{\mu} \omega\right)^{a}, \\
\delta_{0} \omega^{a}=0, & \delta_{1} \omega^{a}=-\frac{1}{2} f^{a b c} \omega_{b} \omega_{c}, \\
\delta_{0} \bar{\omega}_{a}=0, & \delta_{1} \bar{\omega}_{a}=B_{a}, \\
\delta_{0} B^{a}=0, & \delta_{1} B^{a}=0, \\
\delta_{0} \psi_{i}=0, & \delta_{1} \psi_{i}=i \omega^{a} T^{a} \psi_{i}, \\
\delta_{0} \bar{\psi}_{i}=0, & \delta_{1} \bar{\psi}_{i}=i \bar{\psi}_{i} T^{a} \omega_{a}, \\
\delta_{0} \hat{A}_{\mu}^{a *}=0, & \delta_{1} \hat{A}_{\mu}^{a *}=\left.\frac{\delta \Gamma^{(0)}}{\delta A_{\mu}^{a}}\right|_{B_{a}=0, m^{2}=0}, \\
\delta_{0} \hat{\omega}^{a *}=0, & \delta_{1} \hat{\omega}^{a *}=\left.\frac{\delta \Gamma^{(0)}}{\delta \omega_{a}}\right|_{B^{a}=0, m^{2}=0}, \\
\delta_{0} \bar{Y}_{i}=M \bar{\psi}_{i}, & \delta_{1} \bar{Y}_{i}=\left.\frac{\delta \Gamma^{(0)}}{\delta \psi_{i}}\right|_{M=0}, \\
\delta_{0} Y_{i}=M \psi_{i}, & \delta_{1} Y_{i}=-\left.\frac{\delta \Gamma^{(0)}}{\delta \psi_{i}}\right|_{M=0} .
\end{array}
$$

\section{Invariants depending only on $\beta_{V}^{\mu}, \beta_{A}^{\mu}$ with di- mension $\leq 4$}

1. With zero derivatives:

$$
\begin{aligned}
& \Lambda_{V V V V, 1}=\int d^{4} x\left(\beta^{V}\right)^{2}\left(\beta^{V}\right)^{2}, \\
& \Lambda_{A A A A, 1}=\int d^{4} x\left(\beta^{A}\right)^{2}\left(\beta^{A}\right)^{2}, \\
& \Lambda_{A A V V, 1}=\int d^{4} x\left(\beta^{A}\right)^{2}\left(\beta^{V}\right)^{2}, \\
& \Lambda_{A A V V, 2}=\int d^{4} x\left(\beta^{A} \beta^{V}\right)^{2} .
\end{aligned}
$$

2. With one derivative (if at least one $\beta_{A}$ is present we decide not to differentiate $\beta_{V}$ ):

$$
\begin{aligned}
\Lambda_{V V V, 1} & =\int d^{4} x \partial \beta^{V}\left(\beta^{V}\right)^{2}, \\
\Lambda_{V V V, 2} & =\int d^{4} x \partial_{\mu} \beta_{\nu}^{V} \beta^{V \mu} \beta^{V \nu}, \\
\Lambda_{A A V, 1} & =\int d^{4} x \beta^{V} \beta^{A} \partial \beta^{A}, \\
\Lambda_{A A V, 2} & =\int d^{4} x \beta_{\mu}^{V} \beta_{\nu}^{A} \partial^{\mu} \beta^{A \nu}, \\
\Lambda_{A A V, 3} & =\int d^{4} x \beta_{\mu}^{V} \beta_{\nu}^{A} \partial^{\nu} \beta^{A \mu},
\end{aligned}
$$




$$
\Lambda_{A V V}=\int d^{4} x \epsilon^{\mu \nu \rho \sigma} \beta_{\mu}^{A} \beta_{\nu}^{V} \partial_{\rho} \beta_{\sigma}^{V} .
$$

3. With two derivatives:

$$
\begin{aligned}
& \Lambda_{A A, 1}=\int d^{4} x\left(\partial \beta^{A}\right)^{2} \\
& \Lambda_{A A, 2}=\int d^{4} x \partial_{\nu} \beta_{\mu}^{A} \partial^{\nu} \beta^{A \mu} \\
& \Lambda_{A A, 3}=\int d^{4} x \partial_{\nu} \beta_{\mu}^{A} \partial^{\mu} \beta^{A \nu} \\
& \Lambda_{V V, 1}=\int d^{4} x\left(\partial \beta^{V}\right)^{2} \\
& \Lambda_{V V, 2}=\int d^{4} x \partial_{\nu} \beta_{\mu}^{V} \partial^{\nu} \beta^{V \mu} \\
& \Lambda_{V V, 3}=\int d^{4} x \partial_{\nu} \beta_{\mu}^{V} \partial^{\mu} \beta^{V \nu}
\end{aligned}
$$

We denote them by $\int d^{4} x \Lambda_{\beta, i}(x), i=1, \ldots, 16$.

\section{Monomials of dimension $\geq 5$ contributing to the ST breaking terms}

We list the monomials of dimension $\geq 5$ contributing to the ST breaking terms. We use the freedom to perform an integration by parts in order not to differentiate $\bar{\psi}_{i}$ in the ST breaking terms.

$$
\begin{aligned}
& \mathcal{N}_{1}(x)=i \bar{Y}_{i} \not \partial \omega^{a} T^{a} \psi_{i}, \\
& \mathcal{N}_{2}(x)=i \bar{\psi}_{i} T^{a} \not \partial \omega^{a} Y_{i}, \\
& \mathcal{N}_{3}(x)=i \not \partial \bar{\psi}_{i} T^{a} \omega^{a} Y_{i}, \\
& \mathcal{N}_{4}(x)=\bar{Y}_{i} T^{a} f^{a b c} A^{b} \omega^{c} \psi_{i}, \\
& \mathcal{N}_{5}(x)=\bar{\psi}_{i} T^{a} f^{a b c} A^{b} \omega^{c} Y_{i}, \\
& \mathcal{N}_{6}(x)=i \bar{Y}_{i} T^{a} f^{a b c} \omega^{b} \omega^{c} Y_{i} .
\end{aligned}
$$

\section{References}

[1] C. Becchi, A. Rouet and R. Stora, Commun. Math. Phys. 42 (1975) 127.

[2] C. Becchi, A. Rouet and R. Stora, Phys. Lett. B 52 (1974) 344.

[3] C. Becchi, A. Rouet and R. Stora, Annals Phys. 98 (1976) 287.

[4] C. Becchi, "Lectures On The Renormalization Of Gauge Theories," in *Les Houches 1983, Proceedings, Relativity, Groups and Topology, Ii*, 787-821. 
[5] G. Curci and R. Ferrari, Nuovo Cim. A 35 (1976) 273.

[6] T. Kugo and I. Ojima, Phys. Lett. B 73 (1978) 459.

[7] O. Piguet and S. P. Sorella, "Algebraic renormalization: Perturbative renormalization, symmetries and anomalies," Lect. Notes Phys. M28, 1 (1995).

[8] A. A. Slavnov, Theor. Math. Phys. 10 (1972) 99 [Teor. Mat. Fiz. 10 (1972) 153].

[9] J. C. Taylor, Nucl. Phys. B 33 (1971) 436.

[10] S. A. Frolov and A. A. Slavnov, Phys. Lett. B 309 (1993) 344.

[11] T. D. Bakeyev and A. A. Slavnov, Mod. Phys. Lett. A 11 (1996) 1539 arXiv:hep-th/9601092.

[12] J. Gomis, J. Paris and S. Samuel, Phys. Rept. 259 (1995) 1 arXiv:hep-th/9412228.

[13] G. Barnich, F. Brandt and M. Henneaux, Phys. Rept. 338 (2000) 439 arXiv:hep-th/0002245.

[14] For a review see e.g. Refs. [7]-[13].

[15] P. A. Grassi, Nucl. Phys. B 462, 524 (1996) arXiv:hep-th/9505101.

[16] P. A. Grassi, Nucl. Phys. B 560, 499 (1999) arXiv:hep-th/9908188.

[17] E. Kraus, Annals Phys. 262, 155 (1998) arXiv:hep-th/9709154.

[18] W. Hollik, E. Kraus, M. Roth, C. Rupp, K. Sibold and D. Stockinger, Nucl. Phys. B 639, 3 (2002) arXiv:hep-ph/0204350.

[19] R. Ferrari and P. A. Grassi, Phys. Rev. D 60 (1999) 065010 arXiv:hep-th/9807191.

[20] C. P. Martin and D. Sanchez-Ruiz, Nucl. Phys. B 572 (2000) 387 arXiv:hep-th/9905076.

[21] R. Ferrari, P. A. Grassi and A. Quadri, Phys. Lett. B 472 (2000) 346 arXiv:hep-th/9905192.

[22] P. A. Grassi, T. Hurth and M. Steinhauser, Annals Phys. 288 (2001) 197 arXiv:hep-ph/9907426.

[23] P. A. Grassi, T. Hurth and M. Steinhauser, JHEP 0011 (2000) 037 arXiv:hep-ph/0011067.

[24] P. A. Grassi and T. Hurth, in Proc. of the 5th International Symposium on Radiative Corrections (RADCOR 2000) ed. Howard E. Haber, arXiv:hep-ph/0101183. 
[25] P. A. Grassi, T. Hurth and M. Steinhauser, Nucl. Phys. B 610 (2001) 215 arXiv:hep-ph/0102005.

[26] W. Hollik, E. Kraus and D. Stockinger, Eur. Phys. J. C 11 (1999) 365 arXiv:hep-ph/9907393.

[27] W. Hollik, E. Kraus and D. Stockinger, Eur. Phys. J. C 23 (2002) 735 arXiv:hep-ph/0007134.

[28] I. Fischer, W. Hollik, M. Roth and D. Stockinger, Phys. Rev. D 69, 015004 (2004) arXiv:hep-ph/0310191.

[29] J. Zinn-Justin, "Renormalization of gauge theories", SACLAY-D.PH-T74-88, Oct 1974. 40pp. Lectures given at Int. Summer Inst. for Theoretical Physics, Jul 29 - Aug 9, 1974, Bonn, West Germany.Published in Bonn Conf.1974:2.

[30] A. Quadri, JHEP 0304, 017 (2003) arXiv:hep-th/0301211.

[31] W. Bernreuther, R. Bonciani, T. Gehrmann, R. Heinesch, T. Leineweber, P. Mastrolia and E. Remiddi, Nucl. Phys. B 706 (2005) 245 arXiv:hep-ph/0406046.

[32] W. Bernreuther, R. Bonciani, T. Gehrmann, R. Heinesch, T. Leineweber, P. Mastrolia and E. Remiddi, "Two-loop QCD corrections to the heavy quark form factors: Axial vector contributions," arXiv:hep-ph/0412259

[33] S. Aoyama and M. Tonin, Nucl. Phys. B 179 (1981) 293.

[34] M. Tonin, Nucl. Phys. Proc. Suppl. 29BC (1992) 137.

[35] Y. Fujii, N. Ohta and H. Taniguchi, Nucl. Phys. B 177 (1981) 297.

[36] D. Stockinger, "Regularization by Dimensional Reduction: Consistency, Quantum Action Principle, and Supersymmetry," arXiv:hep-ph/0503129

[37] A. Quadri, J. Phys. G 30 (2004) 677 arXiv:hep-th/0309133.

[38] N. N. Bogolyubov and D. V. Shirkov, "Introduction To The Theory Of Quantized Fields," Intersci. Monogr. Phys. Astron. 3 (1959) 1.

[39] K. Hepp, Commun. Math. Phys. 2 (1966) 301.

[40] W. Zimmermann, Commun. Math. Phys. 15 (1969) 208 [Lect. Notes Phys. 558 (2000) 217].

[41] J. H. Lowenstein, Commun. Math. Phys. 47 (1976) 53.

[42] J. H. Lowenstein, "BPHZ Renormalization," NYU-TR11-75 Lectures given at Int. School of Mathematical Physics, Erice, Sicily, Aug 17-31, 1975. 
[43] J. H. Lowenstein and W. Zimmermann, Commun. Math. Phys. 44 (1975) 73 [Lect. Notes Phys. 558 (2000) 310].

[44] J. H. Lowenstein, Nucl. Phys. B 96 (1975) 189.

[45] M. Picariello and A. Quadri, Int. J. Theor. Phys. 41 (2002) 393 arXiv:hep-th/0101167.

[46] A. Quadri, JHEP 0205 (2002) 051 arXiv:hep-th/0201122.

[47] F. Brandt, Commun. Math. Phys. 190 (1997) 459 arXiv:hep-th/9604025.

[48] F. Brandt, Lett. Math. Phys. 55 (2001) 149 arXiv:math-ph/0103006.

[49] P. Breitenlohner and D. Maison, Commun. Math. Phys. 52 (1977) 11.

[50] T. E. Clark and J. H. Lowenstein, Nucl. Phys. B113 (1976) 109.

[51] Y. M. Lam, Phys. Rev. D 6 (1972) 2145.

[52] Y. M. P. Lam, Phys. Rev. D 6 (1972) 2161.

[53] G. Barnich and M. Henneaux, Phys. Rev. Lett. 72 (1994) 1588 arXiv:hep-th/9312206.

[54] G. Barnich, F. Brandt and M. Henneaux, Commun. Math. Phys. 174 (1995) 57 arXiv:hep-th/9405109.

[55] G. Barnich, F. Brandt and M. Henneaux, Commun. Math. Phys. 174 (1995) 93 arXiv:hep-th/9405194.

[56] P. Breitenlohner and D. Maison, Commun. Math. Phys. 52 (1977) 39.

[57] P. Breitenlohner and D. Maison, Commun. Math. Phys. 52 (1977) 55. 